\title{
La inmigración cubana en España. Razones políticas y de sangre en la elección de destino
}

\author{
M. ${ }^{a}$ Pilar González Yanci y M. ${ }^{a}$ José Aguilera Arilla \\ Departamento de Geografía de la UNED
}

\section{RESUMEN}

Los cubanos son uno de los grupos de inmigrantes de más antigua presencia en España, por razones de relación histórica y de la situación política de los últimos años. En el pasado Cuba fue uno de los principales destinos de la emigración de

españoles. Aún hoy, permanecen allí algunos de ellos. La última etapa migratoria que se inicia en 1959 invierte el sentido, Cuba-España, configurándose un grupo de inmigrantes con rasgos diferenciados de otras colonias, en el que destaca el alto número de solicitantes de asilo y de nacionalizaciones. Tras años en que venian como primer eslabón de una migración hacia Estados Unidos, con carácter predominantemente político, en la actualidad, tienden a quedarse en el país, a menudo en situación irregular y cada vez con más presencia de migrantes meramente económicos. En los últimos años las dificultades, tanto de salida de Cuba, como de obtención de visa para entrar en España, están produciendo un nuevo cambio, en el que las cifras se reducen, a pesar de que pervive entre los cubanos el deseo de venir.

\section{ABSTRACT}

Cubans are one of the oldest immigrants groups in Spain, due to their historical relationship as well as to the political situation present over the last few years. In the past, Cuba was one of the main points of destination for Spanish immigrants. Even today, some of them still remain in the island. The last migratory wave, started in 1959, inverts the direction, Cuba-Spain, establishing a group of immigrants with clearly differentiated traits to other colonies, and which distinguished itself by the high number of asylum and nationality seekers. After years in which they arrived as a first stop towards a migration into the United States, with a predominantly political load, they currently tend to stay in this country, often as illegal immigrants and increasingly as migrants for purely economic reasons. In the last few years, the obstacles, both to exit Cuba and to obtain an entry Visa into Spain, are fostering a new change in the trend, with lower figures, even though Cubans still keep their wish of coming to Spain very much alive. 


\section{INTRODUCCIÓN}

Los inmigrantes extranjeros en España son, en la actualidad, relativamente numerosos, no tanto si los comparamos con los países de nuestro entorno cultural, de más antigua tradición inmigratoria (al menos considerada en el siglo veinte), como si lo hacemos con la propia situación española de tan sólo hace tres décadas. Un análisis del conjunto, permite observar la existencia de multitud de contrastes entre unos y otros colectivos y la aparición de similitudes que permiten diferenciar grupos de inmigrantes, por unión de algunas nacionalidades.

Un caso muy particular y diferenciado del resto, es el constituido por los cubanos, que, aunque en algunos aspectos está relacionado con otros grupos, como los hispanoamericanos por su origen y los de Europa del Este, por su antiguo sistema político, presenta, no obstante, claros rasgos de singularidad.

Pese a la proliferación de estudios de la inmigración extranjera en España en los últimos años, no son abundantes los análisis profundos de grupos concretos, salvo algunos que han gozado de especial preferencia, como los marroquíes, portugueses o polacos. El caso de Cuba ha contado con abundante literatura, a menudo considerada como lugar de destino de los emigrantes españoles, al igual que el resto de la América Latina y, en mucha menor medida, también posee algunas monografías de la emigración que se produjo a partir del triunfo de la Revolución Castrista'. En este artículo vamos a hacer una aproximación a la situación actual, no exenta de dificultades.

\section{SINGULARIDAD DE LA COLONIA Y DE LA INMIGRACIÓN CUBANA EN ESPAÑA}

La singularidad de la colonia cubana actual y de los movimientos de cubanos en España se deduce de múltiples hechos:

- Como colonia extranjera registrada y asentada resulta ser "vieja», constituida por familias establecidas en España desde hace bastantes años. Además, representa sólo a una pequeña parte de los cubanos de nacimiento que viven en nuestro país, dado que es muy alto el número de nacionalizados españoles y que, por tanto, están muy integrados y asimilados en nuestra sociedad ${ }^{2}$.

1 En este sentido en 1994 se publicó La emigración cubana en España de C. Martín y V. Romano, en la Fundación de Investigaciones Marxistas, que plantea las características de los emigrantes cubanos desde un planteamiento sociológico, profundizando en los sentimientos y problemas de adaptación de los llegados, en las diferentes etapas que analizan relacionadas con las políticas, acuerdos y desacuerdos entre Cuba, España y Estados Unidos.

2 Para obtener la nacionalidad española, una vez en el país, sólo precisan de dos años de residencia, frente a los diez que se requieren en el caso de otras nacionalidades no latinoamericanas. Además, los hijos de españoles pueden obtener esta nacionalidad en la embajada de España en Cuba. 
- Es una de las migraciones en las que más peso tiene la larga historia que hay detrás, dado que Cuba fue una de las últimas colonias españolas y uno de los principales destinos de la emigración de nuestro país. De modo que el flujo migratorio es, quizás, el más constantemente mantenido entre dos territorios, variando el sentido.

- La inmigración cubana no es hoy el resultado del reciente cambio experimentado en España de país emisor a país receptor. Antes de que este hecho fuera una realidad y simultáneamente a un momento de auge de la salida de españoles hacia Europa, comenzó a llegar, por razones políticas, un flujo de cubanos que puso en España su meta, o su escala, en una emigración de carácter político, que iría evolucionando a lo largo del tiempo.

- En esta migración hay muchas razones «de sangre». Muchos de los que vienen lo hacen porque son de origen español y a veces han mantenido la nacionalidad de sus padres y porque, por ser de carácter político, precisaban de una carta de llamada, fácil de obtener desde nuestro país, donde, en muchos casos, contaban con familiares. España ha supuesto para los cubanos una, y a veces la única, "puerta» de salida hacia el mundo «occidental".

- Existe una marcada dualidad entre los miembros de la "colonia» que está establecida y asentada y a menudo lleva muchos años en España, y los que llegan en los últimos años, entre los que hay una notable precariedad económica que los iguala a los inmigrantes del Tercer Mundo. Dicha dualidad, que también suele darse en otros grupos de inmigrantes, es más acentuada en el caso cubano.

- Es, junto con los inmigrantes del Este, el grupo que más solicita asilo, y uno de los que más busca la nacionalización, esto último fruto de sus relaciones de sangre con nuestro país. Muchos se consideran españoles nacidos en Cuba.

- La mayoría de estas características que adelantamos sobre la singularidad de la migración actual de cubanos, hunden sus raices en la historia de los dos países.

\section{LA EMIGRACION DE ESPAÑOLES A CUBA}

Si en los últimos años la relación migratoria Cuba-España representa un caso muy especial, en el pasado, Cuba era uno de los eslabones de la cadena que unía la vieja metrópoli con el continente Americano. España mantuvo una poderosa corriente migratoria con Cuba, su última colonia americana.

La emigración hacia Cuba es tan antigua que la isla se pobló, tras la desaparición de la población autóctona, por dos corrientes, la africana, formada por emi- 
gración masiva de esclavos, y la española, inducida, orientada, controlada y voluntaria (Iglesias, 1988). En el último cuarto del siglo xIX, hubo medidas políticas encaminadas a fortalecer la presencia de españoles, que tenían sus antecedentes en la Real Cédula de 1817 para establecer colonias de población blanca, y que buscaban desviar a Cuba el exceso de emigración hacia el continente americano y contrarrestar los sentimientos independentistas que afloraban en la isla.

El año 1882, con la creación de un Centro Protector de Inmigrantes y la Ley de Colonización para las Provincias Españolas de Ultramar, se inicia el último gran período de emigración de españoles a Cuba, que conoce el auge y el declive, y que se cierra en 1930, con un epílogo que duró hasta el triunfo de la Revolución, en 1959. Acontecimientos políticos y económicos van marcando sucesivas etapas en este largo período.

Los últimos años de la dominación española, que coinciden con el final del siglo xIX, son años de gran afluencia. Se calcula que se trasladaron a Cuba, aproximadamente, la mitad de los españoles que salieron con destino a América. En 1899 habia cerca de 125.000 españoles en aquella isla, constituyendo su principal colonia extranjera.

La independencia supuso un descenso en el número de emigrantes, que, en un elevado porcentaje, cambió su destino hacia una atractiva Argentina, pero se mantuvo con buen ritmo, de modo que, en 1907 los españoles en Cuba eran un $43 \%$ más que los que había en 1899.

La Primera Guerra Mundial tuvo repercusiones en Cuba, donde se incrementó la necesidad de mano de obra para aumentar la producción de azúcar, ante la mayor demanda de los países en guerra. Se favoreció la entrada de españoles, no sólo para la producción azucarera, sino también para las zonas mineras, con numerosas medidas legislativas, carta de llamada, e incluso con la utilización de agentes reclutadores de emigrantes y campañas de propaganda. Todo lo cual, unido a un descenso del movimiento hacia Argentina, favoreció que el flujo a Cuba no cesara y fuera, entre 1910 y 1925, capaz de absorber la tercera parte de los emigrantes de España a América (Palazón, 1995). En este período se creó la Asociación de Fomento de la Inmigración, para procurar que se llevara a cabo una colonización con familias, y también surgió una Junta de Patronato de Inmigrantes Españoles, para protegerlos de las abusivas condiciones en que se desarrollaba, en ocasiones, la inmigración. 1920 fue un año clave en que emigraron a Cuba cerca de 100.000 españoles, muchos de ellos movidos por el afán de "hacer fortuna".

Pocos años más tarde, en 1925, comenzó la gran recesión económica de Cuba, que llegaría a su clímax con el crack de 1929 y con ella se inició el descenso de emigrantes españoles $y$ un proceso mediante el cual este país iba a sufrir una transformación. De ser destino de población se convirtió en lugar de origen de la emigración. La colonia española en Cuba decreció en 1930 con respecto al pasado y ya nunca se recuperaría. En esta fecha estaban censados un total de 257.596 españoles en la isla. 
A lo largo de todos estos años, 1882-1930, la emigración española estuvo constituida por contingentes de predominio masculino, jóvenes, activos, que acudían, sobre todo, a trabajar en la agricultura y en el comercio, y que, en una parte importante, eran emigrantes de carácter estacional, de modo que constantemente hubo un flujo de retorno, habiendo, no obstante, un saldo negativo para España, que perdería en torno a 300.000 habitantes en el período.

Hubo una mayoria de gallegos, asturianos y canarios en casi todos los momentos, que se concentraban en La Habana y provincias como Matanzas y Las Villas, aunque con los años, se fueron incrementando, sobre todo en las provincias del este, hacia donde avanzaba la explotación azucarera.

El retorno, que fue habitual por ser una emigración de tipo estacional, a partir de 1925 tuvo otro carácter, una repatriación de españoles que quedaban sin trabajo ${ }^{3}$.

La Guerra Civil Española supuso una considerable salida de españoles al exilio. Uno de los destinos fue Cuba. Muchos partieron hacia Francia, donde algunos se naturalizaban cubanos y eran repatriados a Cuba tras obtener pasaporte y visado. No obstante, no fue un número muy elevado. Hubo un grupo de intelectuales y algunos cientos de reemigrados que en una primera fase fueron a Santo Domingo, de donde salieron en el año 1945 perseguidos por la dictadura de Trujillo. Es también de destacar, que muchos de los que fueron a Cuba, en realidad utilizaron este destino como etapa de su viaje a Méjico, en una situación que se parece a lo que va a ocurrir después, cuando, como veremos, los cubanos que salen con el régimen castrista, acuden a España para luego saltar a Estados Unidos. Los que quedaron en Cuba, sin permiso de entrada en Méjico, tuvieron que soportar condiciones difíciles, ya que habia poco trabajo y una legislación de férreo control del mercado laboral y protección de los nacionales. Sobrevivieron gracias a organismos como el Círculo Republicano Español.

La corriente española a Cuba entre 1946 y 1958 fue débil y en franco descenso. No llegaron a 20.000 los emigrados, mientras que el retorno era considerable (13.588 en el período) (Palazón, 1995).

El triunfo de la Revolución de Castro supuso prácticamente el fin de la emigración de España a Cuba. Entre 1959 y 1967 salieron con este destino 1.109 españoles y entre 1968 y 1990, 112, siendo ésta la tónica actual. Entre 1985 y 1994 se contabilizan 97 emigrantes españoles a Cuba, de los cuales 65 eran hombres y 32 mujeres y entre 1995 y 2001 tan sólo 17. Resulta, por tanto, una cifra meramente testimonial, en un proceso de descenso generalizado de la emigración española, que, en el caso concreto del continente americano pasó de 1.581 personas en 1992 a 47 en 2001 (Anuario de Migraciones 2002).

${ }^{3}$ La Ley del $50 \%$, que obligaba a que por cada extranjero trabajando en una empresa hubiera al menos un cubano, deja a muchos españoles sin trabajo y mueve a buscar la nacionalización cubana a otros. En 1933 hubo incluso un decreto que obligaba a la repatriación de los extranjeros desocupados y en 1937 se obligaba a los emigrantes a efectuar un depósito de una cantidad de dólares al entrar al país, para garantizar la repatriación. 
Incluso son ya pocos los españoles que quedan en Cuba, que en buena medida son nacidos alli, pero que han conservado la nacionalidad de sus padres. Según datos de la Dirección General de Migraciones (Anuario de Migraciones, 1995) en 1970 quedaban 74.026 españoles, que en 1980 descendieron a 60.000 , mientras que en 1994 sólo se registran 1.184. La salida del país y los fallecimientos de los más ancianos explicarían este descenso, sin que debamos olvidar que las estadísticas no son del todo exactas. En 1999 los españoles residentes en Cuba eran 15.775. Después de esta fecha, según los datos del último anuario de migraciones (2002) han ido en aumento, alcanzando en 2001 la cifra de 20.411. Sin duda, este nuevo incremento está motivado por las nuevas relaciones establecidas por algunas empresas españolas en Cuba, como hoteles, bancos, etc. También resulta significativo el número de pensiones asistenciales, por ancianidad, que se siguen pagando a españoles residentes en la isla. En 1994 el gobierno español abonaba pensión a 1.100 personas, que, en 1995, ascienden a 2.906 y en 2001 a 3.103. En este caso, el incremento ha de estar justificado por el envejecimiento de la población que permanece en Cuba de los antiguos emigrantes españoles. Las pensiones pagadas a argentinos y venezolanos suponen, junto a las de los cubanos, las de los tres grupos más numerosos en el extranjero.

\section{LA INMIGRACION CUBANA EN ESPAÑA}

$\mathrm{Si}$, como hemos apuntado, siempre hubo un flujo de retorno de españoles, por el carácter estacional de buena parte de la emigración española a Cuba, a partir de 1959 se inicia un nuevo periodo en el que la movilidad se da casi exclusivamente en un sentido, de Cuba a España.

Los acontecimientos históricos que tuvieron lugar en Cuba en la década de los 50, culminaron en 1959 con la caída de Batista y el triunfo de la Revolución Castrista. A partir de ese momento, comienza a llegar a España un flujo migratorio cubano muy distinto al que lo hacía en etapas anteriores. Ya no son sólo retornados temporales, sino que son personas de antiguo asentamiento, que, debido a las nuevas circunstancias políticas y económicas, se ven obligadas o desean abandonar la isla en la que residían. Esta corriente migratoria presenta unas características peculiares, basadas, precisamente, en su origen español, en los lazos de sangre que la unían con España. Los inmigrantes que llegan a nuestro país desde Cuba, sobre todo en los primeros momentos, suelen ser españoles (de nacimiento o de nacionalidad) o hijos o nietos de españoles.

Aunque la principal motivación, denominador común de las causas expresadas por este grupo al salir de su país, es la disidencia política, se pueden establecer diferencias en el grupo de emigrantes cubanos en el extranjero, por la situación económica personal y familiar y el momento o etapa en la que dejan Cuba. 
Gracias a los testimonios de protagonistas de la nueva experiencia emigratoria cubana desde $1959^{4}$, hemos podido establecer algunas etapas diferentes dentro del período de 45 años que constituye la historia reciente de Cuba.

En una primera etapa, que iría desde 1959 hasta mediados de los 70, abandonaron la isla más de un millón de personas que tenían como destino último los Estados Unidos. Muchos de ellos, en torno a 300.000 , salieron de Cuba hacia España como primer destino, gracias a las relaciones de sangre que existían entre ambos países y a la buena acogida de un gobierno contrario al régimen de Castro. En estos momentos la salida era relativamente fácil, si la comparamos con otros posteriores.

El caso de los españoles residentes en Cuba, o de sus hijos nacidos o no en la isla, supone, en los primeros momentos, un elevado contingente de población que llega a nuestro país con el deseo de retornar a su tierra o a la de sus mayores. Muchos de ellos se fueron después a los Estados Unidos, pero otros muchos se quedaron y dada su nacionalidad española, antigua o modernamente adquirida, no aparecen en las estadísticas oficiales de inmigrantes.

De 1960 a 1965 suelen salir familias completas, pero, desde finales de los 60, comienzan a surgir mayores dificultades, debido al endurecimiento de la ley, que obligaba a los hombres a estar dispuestos a servir a su patria desde los 15 hasta los 40 años. Esto hace que se dificulte aún más la salida y por ello dejan de ser ya familias enteras las que abandonan el país, e incluso, muchas veces son niños solos los que lo hacen. Inspirados en los barcos de niños que desde España viajaran hasta Rusia, en la Guerra Civil Española, se organizó, entre 1960 y 1965, la operación Pedro Pan (Peter Pan) que se materializó en 1965 con la llegada de barcos con niños de origen español, a los que se acogia en el albergue, acondicionado para ello, de la Casa de Campo de Madrid, y más tarde, en El Escorial. Muchos de estos niños viajaron también posteriormente hacia los Estados Unidos. En esta operación se calcula que salieron 14.000 niños solos, 24.000 acompañados y 48.000 adultos, gran parte de ellos de forma clandestina ${ }^{5}$. El sistema para salir de Cuba, en esta primera etapa, se fue endureciendo con el tiempo. El emigrante cubano debía de cumplir con una serie de requisitos impuestos por el gobierno. En primer lugar, presentar una carta en la que se le reclamase desde el exterior por un familiar, lógicamente España tenía un gran número de ellos, lo que privilegiaba su posición respecto a esta exigencia frente a otros países. El familiar debía enviar un dinero, en el caso de España vía Canadá, para el pago del pasaje. El residente en la isla había de renunciar, además, a su trabajo, negocio, propiedades, etc. y dos días antes de salir, abandonar su vivienda, pues tenía que ser todo inventariado y en algunos casos, pasar a un campo de trabajo hasta su salida definitiva del país.

4 Agradecemos especialmente la colaboración de D. Antonio Peláez Huerta, historiador del Centro Cubano en España (Madrid) y a la secretaria del centro, Dña María Comella.

5 Según los autores del libro La emigración cubana en España, antes citado, esta operación fue organizada por la Agencia Central de Inteligencia estadounidense. 
A partir de principios de los setenta, la emigración comienza a declinar. Existía un Comité Internacional de Rescate que ayudaba a la salida, pero el gobierno aumentó las restricciones a la misma. Desde principios de los setenta hasta 1980, la ayuda de los cubanos residentes en La Florida fue muy importante para este grupo de emigrantes que salieron en circunstancias económicas mucho peores que las de sus compatriotas en fechas anteriores. En esta etapa en los deseos de salir, la motivación económica se había sumado a la común y más generalizada del ideario político, a pesar de lo cual, como señalábamos antes, el número se redujo.

En 1981, tras el incidente de los cubanos refugiados en la Embajada del Perú, hubo una importante salida que contó con el apoyo de los residentes en Miami. Este año marca el comienzo de la última etapa que llega hasta el presente y que se caracteriza por un aumento paulatino del número de inmigrantes, aunque ya moviéndonos en cifras muy discretas, y por la presencia de rasgos muy diferentes de los del pasado.

\section{ANÁLISIS DE LA SITUACION A FINALES DEL SIGLOXX}

A finales del siglo $x x$, la situación de los inmigrantes cubanos en España presentaba similares dificultades de análisis a las de cualquier otro grupo, por las propias características de la movilidad de esta población. La información que nos proporciona el Censo de 1991 es parcial, pero interesante. En él se ponía de relieve que, habiendo más de 25.000 personas nacidas en Cuba, solamente eran extranjeros (de nacionalidad no española) 3.539 nacidos en Cuba y 3.391 de nacionalidad cubana, lo que indica el gran número de cubanos que habian obtenido, o que mantenían por su origen, la nacionalidad española?.

La pirámide de edades de la colonia cubana (considerando así a los que poseen esta nacionalidad) presentaba claros rasgos de envejecimiento. El porcentaje de personas de más de 65 años era muy superior al que presentaba la población española e, incluso, mayor que los de otras colonias de inmigrantes, como las de los europeos occidentales, que eran, entre los extranjeros, los más envejecidos, contrastando con los procedentes de otros países hispanoamericanos, con los que tienen evidente relación, que, en cambio, eran mucho más jóvenes. Esa elevada proporción de ancianos explica también la numerosa presencia de viudos (casi un $15 \%$ ). Si a ello sumamos la escasez de niños, sobre todo en los primeros años de vida, los rasgos de envejecimiento de este grupo eran todavía más acentuados que

6 En el citado libro de C. Martín y V. Romano se marcan unas etapas ligeramente diferentes. La primera de las aquí consideradas está subdividida en tres, de 1959 a 1962 la primera, al romperse las relaciones con Estados Unidos, tras el conflicto de bahia Cochinos; la segunda de 1962 a 1965, al reestablecerse el vuelo directo entre la isla y USA y desde este momento hasta 1973 la tercera etapa.

7 Los cubanos son unos de los colectivos que mayor número de nacionalizaciones alcanza cada año. En 1983 totalizaban 376 que suponia el 7\% del total de concesiones en aquel año. El número ha ido descendiendo, oscilando desde 1995 hasta la actualidad en alrededor del $2 \%$. 
los de cualquier otro colectivo, tanto español como extranjero. El envejecimiento puede también reflejar que habría mayor proporción de jóvenes que se nacionalizaban españoles.

Los cubanos censados en 1991 tenían, fundamentalmente, un nivel de estudios medio. La proporción de analfabetos y sin estudios (15,5\% del total) se compensaba con los que, por el contrario, poseían el nivel más alto $(15,1 \%$ con tercer grado).

Había un elevado porcentaje de inactivos, relacionado con la edad antes comentada y con el hecho de que las mujeres eran mayoritariamente jubiladas y amas de casa. La tasa de paro era también muy alta $(28,7 \%)$ y algo superior en el caso de las mujeres. En la situación profesional destacaba el hecho de la considerable proporción de empresarios (un $7,1 \%$ con asalariados a su cargo y un $18 \%$ sin ellos). La profesión muestra una buena situación, dado que el $25 \%$ eran profesionales, técnicos y directivos, mientras que el grupo de peones y trabajadores sin especializar sólo suponía un 4,6\%. La mayoría estaban incluidos en el sector terciario, destacando la actividad en el comercio.

A pesar de que las mayores cifras de llegada de cubanos a España son de los años 60-75 (más de 300.000) con motivo del triunfo de la Revolución, los censados en 1991 han llegado sobre todo a partir de 1980, coincidiendo con el momento en que empieza a manifestarse en nuestro país la afluencia importante de extranjeros, dado que aquellos contingentes iniciales en parte estaban nacionalizados, y sobre todo, en su mayoría, se trasladaron a un tercer destino, normalmente Estados Unidos.

Antes de 1960 llegó un $23,6 \%$, mientras que entre el 80 y el 90 lo hicieron casi el $51 \%$. Dentro de la década, aparte del año 1981, en que llegó un número mayor, como consecuencia del envío al exterior de los refugiados en la Embajada del Perú en La Habana, se produce un aumento del volumen de llegadas en 1987 y siguientes, coincidiendo con un auge de la economia española. La distribución espacial en España de estos últimos llegados (desde 1980) era de concentración en la capital $(44 \%)$ seguida de las provincias Canarias (16\%) Barcelona $(8.9 \%)$ y la suma de las provincias gallegas $(6 \%)$.

El total de censados no era coincidente con la cifra de residentes que se obtiene a partir de las estadísticas de la Dirección General de la Policía. En el caso de los cubanos la cifra de residentes, considerando los últimos 25 años del siglo, es oscilante, pero con una clara tendencia al descenso hasta 1992, año en el que vuelve de nuevo a ascender.

Los datos de la Dirección General de Migraciones, que sólo son disponibles desde 1985, muestran algunos detalles de los contingentes que llegaban cada año. Lo primero que se ha de destacar es que en este grupo de inmigrantes hay españoles y extranjeros. Los primeros eran "retornados» pero en su gran mayoría habian nacido en Cuba y suponían en torno a un $30 \%$ de todos los que llegaban. Hay 
un cierto predominio de mujeres, aunque varía según los años, siendo más numerosos en los últimos. En general estaban bastante equilibrados por sexo.

Llegaban de todas las edades, pero con un predominio apreciable de los jóvenes, por debajo de 40 años y en especial en el grupo de 25 a 34 . El nivel de estudios era bastante elevado, predominando los que tenían bachiller y estudios superiores. Se distribuían por las Comunidades Autónomas Españolas con una clara concentración en la de Madrid y en las comunidades de las que procedían la mayor parte de los emigrantes que fueron a Cuba en el pasado: Canarias, Asturias y Galicia. Dentro de éstas se instalaban preferentemente en las capitales provinciales y en ciudades de más de 100.000 habitantes, aunque sin olvidar que muchos deseaban volver a la aldea o pueblo que les vio nacer y se dirigian, aunque en número más reducido, a municipios pequeños, de menos de 10.000 habitantes, donde vivieron ellos o sus antepasados.

Tras este somero repaso a las características que nos muestran las estadísticas oficiales, podemos añadir, a través de la información oral obtenida de algunos de los cubanos que, recién llegados, acudían al Centro Cubano de Madrid y de los testimonios de antiguos inmigrantes y estudiosos del proceso, que los cubanos que a finales del siglo llegaban a España eran de diferentes tipos. Seguía habiendo retornados, 677 según las bajas consulares entre 1987 y 1994, que naturalmente seguian siendo españoles. Por otro lado, llegaban artistas, que venían a trabajar y que, con alguna frecuencia, una vez en el país, solicitaban quedarse como asilados políticos. Otro grupo eran los profesionales que venían a realizar cursos de formación en empresas que tienen relación con Cuba. También entre éstos se daban casos de algunos que solicitaban asilo político. Había estudiantes, amparados en un programa de cooperación y, por último, los verdaderos inmigrantes que llegaban tras haber solicitado en Cuba un visado de salida a España, que obtenían con gran dificultad, o bien utilizaban la carta de invitación, a veces falsa y comprada, de un familiar, de España o de algún país del Este, para el que no precisaban visado. Una vez que se encontraban en España, en el aeropuerto de Madrid, buscaban la forma de obtener el estatuto de asilo ${ }^{8}$ y se quedaban en el país, algunos acogidos en los albergues para extranjeros, otros en Asociaciones Católicas que les facilitaban asistencia, muchas veces a través de Centro Cubano. En realidad, lo que a menudo ocurría es que no venían a España como meta final de su emigración, pues poco tiempo después desaparecían, pasando, incluso de forma irregular, a los Estados Unidos, donde dada la gran colonia de cubanos existente, encontraban ayuda y la posibilidad de un trabajo. (En buena medida el dinero para

${ }^{8}$ Los cubanos son uno de los colectivos que en mayor proporción solicitan acogerse al estatuto de asilo. En el pasado lo conseguían con facilidad, obteniendo numerosas ayudas, incluso económicas. Desde la entrada de España en la Unión Europea y con la nueva Ley de Asilo, es mucho más difícil de obtener. No obstante, entre 1985 y 1995 se les concedió a 671 solicitantes, afectando a 1086 personas. En la actualidad resulta muy difícil. Además, con frecuencia ni siquiera lo pueden pedir en España, porque llegan antes a otro pais de Europa y, según la ley el asilo sólo se puede solicitar en el primer pais en el que entran. 
el pasaje a España procedía de amigos o familiares que residian en aquel país). España era el vértice del triángulo que unía Cuba con Estados Unidos, meta verdadera de su emigración. Los que llegaban por estos procedimientos eran mayoritariamente jóvenes, venían solos, dejando atrás la familia, con afán de empezar una nueva vida. Eran muy diferentes a los primeros que salieron en las etapas antes descritas, la mayoría habían nacido y se habían formado en el régimen político actual. Argumentaban motivos políticos, sobre todo su deseo de obtener libertades, pero en el fondo es palpable el gran peso de la motivación económica.

Lo hasta aquí expuesto, debidamente actualizado, corresponde al análisis de los movimientos migratorios entre Cuba y España, que presentamos al VI Congreso de la Población, celebrado en Huelva en 1997 y que permanecía inédito, al no llegar a publicarse las correspondientes actas de la reunión. Por este motivo se publica aquí como artículo, al que añadimos un capítulo nuevo en el que se analiza la situación actual, en 2004, valorando los cambios que se han producido.

\section{SITUACIÓN ACTUAL}

En los años transcurridos desde 1991 hasta la actualidad, la inmigración de extranjeros en España ha sufrido una notable aceleración, llegando a duplicarse su número entre los dos censos, en las cifras oficiales, que, no obstante, ocultan el elevado volumen de irregulares que, a pesar de los procesos de regularización, han seguido incrementándose.

A partir de los datos del censo de 2001 y los anuarios de migraciones y de extranjería, podemos obtener una buena aproximación a la situación actual de la inmigración cubana, al menos de la residente legalmente.

En el 2001, según el Censo de Población y Vivienda, vivían en España 50.765 personas nacidas en Cuba, de las cuales, 24.513 poseian la nacionalidad española, confirmando el fuerte arraigo de esta población en el pais. El número de cubanos de nacionalidad, que poseían permiso de residencia, era de 21.467, que son los que tienen la condición de inmigrante propiamente dicha.

Atendiendo a este grupo, en los años transcurridos desde 1995 se aprecia un constante incremento del número de llegados, que pasa de 6.097 a 27.323 a finales de 2003, en proporción similar a la del conjunto de extranjeros en España, que se cuadruplica en estos años. La fecha de llegada que recoge el censo de 2001 muestra lo reciente de la presencia de muchos de ellos en el país. De los 25.613 censados sólo 1.253 llegaron antes de 1961, mientras que el $67 \%$ llegó entre 1995 y 2001.

El nuevo grupo de inmigrantes cubanos se caracteriza por la estructura siguiente. Un predominio de mujeres $(57,4 \%$ ) y de adultos, de entre 25 y 40 años, como se observa en la pirámide de edades correspondiente (Figura 1). Su distribución espacial es por todo el territorio, mostrando más relación con la distribución 


\begin{tabular}{|l|l|}
\hline Ambito geogrático: & Nacional \\
\hline Coleclivo: & Extranjeros \\
\hline Unidad de medida: & Personas \\
\hline Filtros: & Pais de nacionalidad Cuba \\
\hline
\end{tabular}

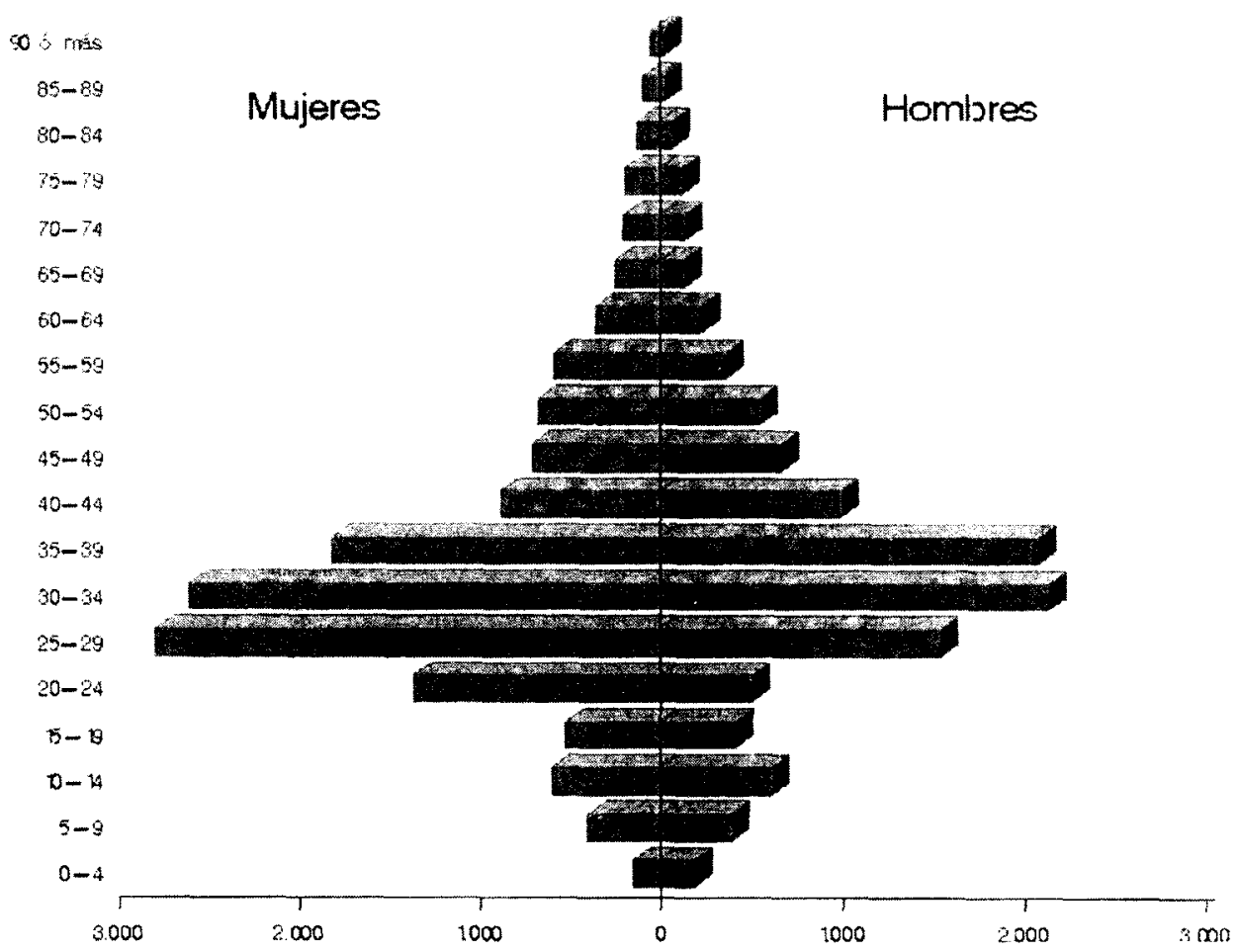

Figura 1. Pirámide de la población de residentes extranjeros de nacionalidad cubana en España. Fuente INE. Censo de la Población y Vivienda 2001.

del actual volumen de población española por comunidades, que con las antiguas regiones de las que salieron en el pasado, mayoritariamente, los españoles hacia Cuba. La comunidad actual con más inmigrantes cubanos es Canarias, seguida de Madrid y Cataluña (Figura 2).

Los cubanos siguen siendo de los grupos que en mayor proporción solicitan asilo político, dadas las circunstancias que concurren en su país. En 2002 fueron la segunda nacionalidad solicitante de asilo $(18,7 \%)$ tras Nigeria (Figura 3 ) aunque en 2003 ha disminuido notablemente el número de solicitantes respecto a años anteriores. En cambio, las nacionalizaciones son muy numerosas. Entre 1991 y 2001 se multiplica por mil el número de cubanos que obtienen la nacionalidad española, de forma similar a lo que ocurre en algunos otros países de América Latina (República Dominicana y Perú). 


\begin{tabular}{|l|l|}
\hline Amblto geogranco: & Nacional \\
\hline Colectho: & Extranjeros \\
\hline Unided de medida: & Porsonas \\
\hline Flltros: & Pois de nacionalidad =Cuba \\
\hline Variables no geogrifficas: & \\
\hline
\end{tabular}
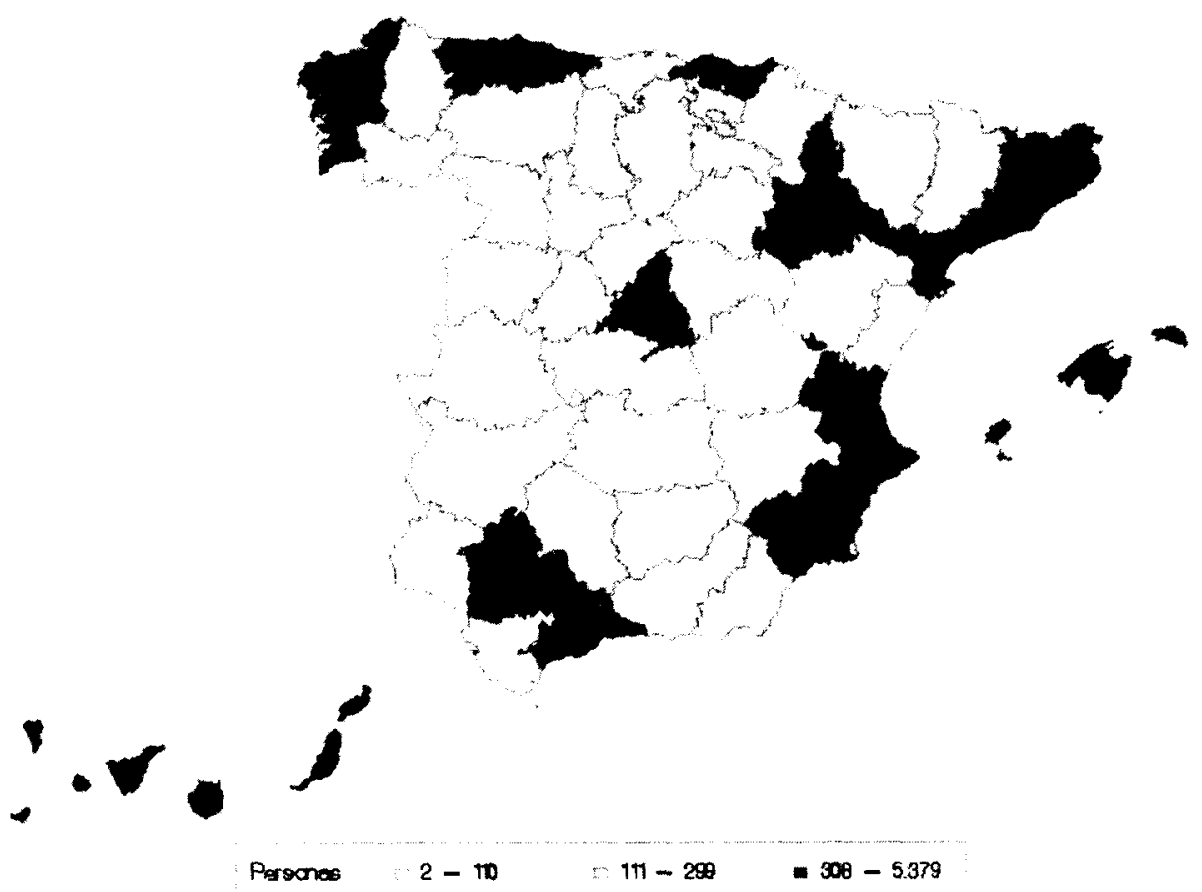

Figura 2. Distribución de los inmigrantes cubanos en España.

Fuente: INE. Censo de Población y viviendas 2001.

A pesar de que cada vez son más similares a los restantes migrantes económicos, los cubanos mantienen, como los argentinos, un mayor nivel cultural, como lo demuestra el que sea uno de los colectivos que en mayor medida solicita la homologación de títulos universitarios (463 solicitudes en 2003) sólo superadas por Argentina, Colombia, Italia y Perú. Incluso entre ellos una parte son estudiantes con autorización de estancia (232 en 2003), en este caso con predominio de varones $(58 \%)$. También son relativamente numerosos los que se encuentran realizando estudios universitarios de segundo y tercer ciclo. Igualmente, es un indicador de la superioridad en la formación el hecho de que en el régimen de cotización a la seguridad social el $9,3 \%$ sean ingenieros y licenciados.

A comienzos de 2004 el $54 \%$ eran activos, con diferentes situaciones laborales, la mayoría trabajando por cuenta ajena y el $8 \%$ por cuenta propia, en los diferentes regímenes de la Seguridad Social, la gran mayoría en el régimen general y con 


\section{Solicitantes de asilo. 2002.}

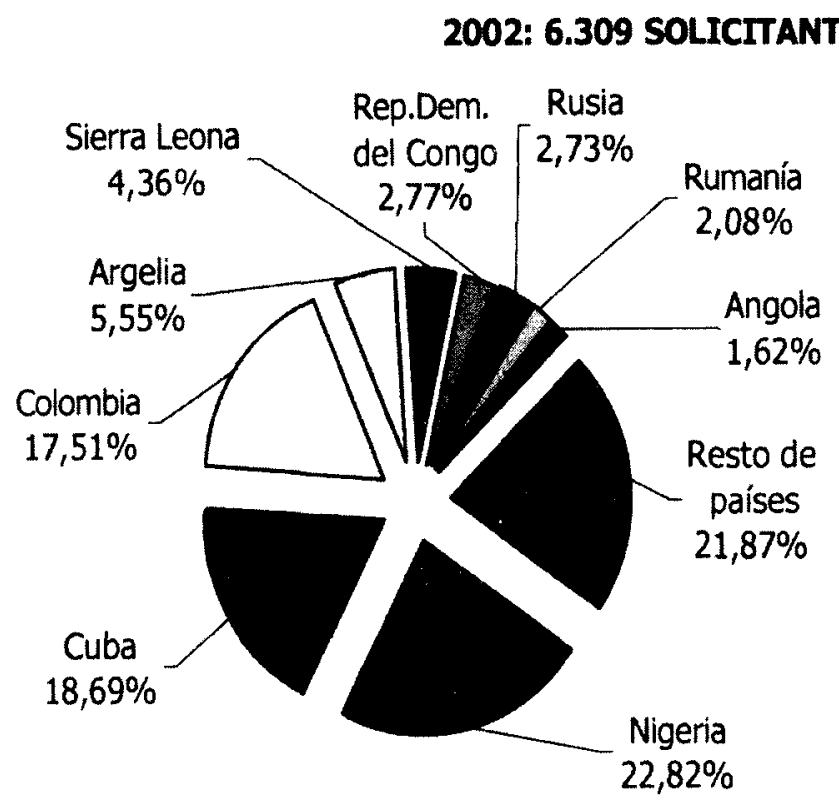

Figura 3. Solicitantes de asilo según procedencia, en España. Fuente: INE. Censo de Población y viviendas 2001.

contratos indefinidos, en un $40 \%$, pero también en algunos de los regímenes especiales, como el de empleadas de hogar, que acoge a un 3,24 \% de los trabajadores. En los primeros años de la liegada de cubanos a España era raro que se ocuparan en estos trabajos. Incluso, tal como veíamos en la primera parte, hasta 1991 habia, entre los cubanos, un predominio de profesionales, técnicos y directivos, un $25 \%$, frente a un $4,6 \%$ de obreros sin especializar. Actualmente, en cambio, el porcentaje de peones alcanza el $23,02 \%$, mientras que sólo son directivos y técnicos en torno a un $12 \%$.

Todo ello confirma que el colectivo de cubanos cada vez tiene más carácter de migración económica, frente a su anterior situación más relacionada con circunstancias políticas.

De la comparación de los dos momentos censales, 1991-2001, se desprenden algunos notables cambios en las características de la inmigración cubana, a pesar de que la situación de origen no ha variado en lo fundamental. Sí lo han hecho, en cambio, determinadas circunstancias que han afectado a la economía cubana y a las posibilidades de entrada a algunos países. Hasta el año 2002 el volumen de cu- 
banos que venía a España fue en aumento, con un máximo en 2001. A partir de este año se ha iniciado un descenso como nunca se habia conocido antes. En el Centro Cubano, a donde acuden muchos de los llegados a España, o cuanto menos a Madrid, el número de atendidos pasó de 1.100 en 2001 a 60 ó 70 en 2004 (datos aún provisionales). Las razones de este último descenso son varias. España no concede en la actualidad visa de entrada a los solicitantes en Cuba, donde, por otro lado siguen siendo grandes las dificultades para marcharse. Continúan permitiendo la salida, previa presentación de una carta de invitación desde el exterior, pero no conceden el permiso a los titulados (el trámite para lo que llaman la “liberación» es de cinco años para estas personas.

No obstante, son aún muchos los cubanos que acuden a España, controlados o no. Entre éstos, dejando a un lado a los que están con permiso de estudios, un buen nümero son funcionarios del estado, de niveles medios, que son enviados a diversos países de Europa, para realizar determinadas misiones, que les son encomendadas o para hacer cursos de formación, en este caso incluso por parte de algunas de las empresas españolas destacadas en la isla (hoteles, bancos, etc.). Una vez en este continente deciden quedarse y piden asilo, lo cual es dificil de conseguir. En los últimos tiempos han optado por este sistema atletas, deportistas, artistas y funcionarios de no muy alto nivel, además de un cierto número de médicos, que fueron enviados por el gobierno a África y han abandonado su misión, sin desear regresar al país. Según información del Centro Cubano, entre los enviados fuera de Cuba se genera gran descontento porque les es retenida una parte importante de los sueldos percibidos, que suelen estar abonados por el país de acogida, lo que les obliga a vivir con dificultades económicas. Este es uno de los motivos, al parecer, de que intenten quedarse en los nuevos países, solicitando asilo o, incluso, como irregulares, dispuestos a aceptar cualquier trabajo.

La presencia de compatriotas en muchos países de Europa, dadas las antiguas relaciones de Cuba con los países del Este, facilita el envío de cartas de llamada, vía muy utilizada para salir del país, aunque un buen número de los que por este método acceden a países europeos tratan luego de entrar en España. Es incluso frecuente que si antes de llegar al país del que procede la carta de llamada, el vuelo realiza escala en España, aprovechen allí mismo para solicitar el asilo político. En los demás casos, lo piden en el país europeo al que llegan. A menudo, las circunstancias económicas y políticas de aquéllos, además de las diferencias culturales, idiomáticas y hasta climáticas, les hacen desistir de permanecer allí e intentar atravesar las fronteras, hoy abiertas de Europa, hasta llegar a España, donde ya no pueden solicitar asilo, por no ser el primer destino, optando por permanecer en situación irregular. Camino que han de recorrer sorteando no pocas dificultades, siguiendo las mismas vias que otros europeos, que desde los antiguos países del

9 En los últimos años se han endurecido los requisitos exigidos para conceder el asilo, siendo necesario demostrar de forma fehaciente que se es contrario al régimen imperante o que se está perseguido por razones políticas, de homosexualidad, religiosas, etc. 
Este también llegan en situación irregular. Una vez en España se insertan con cierta facilidad en la población y consiguen trabajos, cada vez, eso si, menos cualificados. Hasta el 2001 tras la estancia en España saltaban a Estados Unidos, de forma bastante generalizada (el $90 \%$ de los que acudían al Centro Cubano de Madrid). Hoy, en cambio, en parte como consecuencia de los acontecimientos terroristas de septiembre de 2002, la frontera americana está herméticamente cerrada a los inmigrantes.

La peripecia del cubano para salir de su país y llegar a España no ha variado mucho, pero si lo ha hecho el perfil de los emigrantes, que ya no son tanto disidentes políticos, sino sobre todo emigrantes económicos, que quieren buscar oportunidades fuera y mejorar su situación, con muy poco conocimiento del exterior, por lo que, cuando se encuentran en Europa pasan por momentos difíciles hasta lograr cierta estabilidad.

Una diferencia con otros inmigrantes es el apoyo que encuentran en España, aún hoy, pero sobre todo en los años pasados, por parte de centros de acogida (el citado Centro Cubano es el principal ejemplo 19 y por el hecho de que en España está instalada una importante colonia de cubanos que vinieron, estos sí mayoritariamente, como disidentes desde el comienzo de la Revolución, además de contar, incluso, con familiares por el origen español de muchísimos de ellos.

\section{CONCLUSIÓN}

La presencia de cubanos en España y de españoles en Cuba ha sido tradicional y muy numerosa, a pesar de los pequeños contingentes que encontramos en la actualidad en España, debido, además de a las masivas nacionalizaciones 0 al mantenimiento de la nacionalidad española en muchos casos, a que nuestro país ha sido, para una inmensa mayoría, un lugar de paso hacia su destino más deseado, Estados Unidos. Hoy ya no está abierta esta puerta y además no resultan fáciles las condiciones de entrada en España por lo que los cubanos que llegan, a menudo desde un país europeo del Este, tienden a permanecer, muchas veces en situación irregular, ante las dificultades de obtener el asilo.

En los últimos años, en que, tras un incremento de las llegadas se vuelve a un descenso drástico, el cambio más significativo está en el perfil del inmigrante, alejado del que tenía antiguamente y cada vez más coincidente con el de los migrantes meramente económicos.

La historia, las relaciones familiares, la situación política y la formación de un triángulo migratorio entre España y un destino anterior o posterior, confiere su originalidad a este grupo de emigrantes.

${ }^{10}$ Eran numerosos los organismos, tanto oficiales, del gobierno de Franco, como internacionales y de la Iglesia Católica, algunos, naturalmente con carácter político 


\section{BIBLIOGRAFÍA}

anuario Estadistico de Extranjería. Dirección General de Migraciones. Varios años.

ANuARIO de MigraciónEs. Dirección General de Migraciones. Varios años.

Censos de la Población y Vivienda de España 1991 y 2001. Instituto Nacional de Estadistica.

GAVIRA, C. Dominación colonial y espacio social. Ciclos productivos, estructuras de dominacióny pautas de asentamiento. Cuba, siglos XVI al XX. Tesis doctoral. Universidad Complutense de Madrid. 1976.

Historia 16: Historia General de la Emigración Española a lberoamérica. 1992. tomo 1. 732 pp. tomo 2. 550 pp. + fotos

IGLESIAS GARCiA, F.(1988): "Características de la inmigración española a Cuba. 1904-1930». En : Sánchez Albornoz, N.: Españoles hacia América, la emigración en masa, 1880-1930. Madrid. Alianza Editorial. pp. 270-295.

MARTín FERnÁndez, Consuelo (1994): La emigración cubana en España. Madrid Fundación de Investigaciones Marxistas. 110 pp.

NARANJO, C. (1984): “Análisis Histórico de la emigración española a Cuba 1900-1959». En Revista de Indias 1984, vol. XLIV, n. ${ }^{\circ} 174$. pp. 505-528.

PALAzÓN FERAANDO, Salvador, (1993): “La emigración española a Latinoamérica. 1946-1990. Reanudación y crisis en el flujo secular». Estudios geográficos. n. 210. pp. 97- 128.

- (1995): «Capital humano español y desarrollo económico latinoamericano. Evolución, causas y caracteristicas del flujo migratorio. (1882-1990). Diputación de Alicante. pp. 405.

- (1995): “Los españoles en América (1850-1990)". CEDEAL. Madrid. 270 pp. 